\title{
Michel Accad. (2017). Moving Mountains: A Socratic Challenge to the Theory and Practice of Population Medicine. Huntsville, TX: Green Publishing House. ISBN 978-1-63432-030-6. A Dichotomization of Health?
}

\author{
Peter C Wyer MD \\ Associate Professor of Medicine, Columbia University Medical Center, New York, NY, USA
}

\begin{abstract}
Keywords
Clinical outcomes, dichotomization of health, disease, epidemiology, evidence-based medicine, health, hypertension, person-centered healthcare, personhood, population health, population medicine, public health
\end{abstract}

\section{Correspondence address}

Dr. Peter Wyer, Department of Emergency Medicine, Columbia University, 622 W $168^{\text {th }}$ Street, New York, NY 10032 , USA. E-mail: pw91@cumc.columbia.edu

Accepted for publication: 17 January 2018

\section{Introduction}

Within today's healthcare system, conflicting priorities and the varying perspectives of patients and providers are the leading drivers of complexity. The health of populations and the care of individual patients define two such perspectives and are potentially in conflict. Because each of these perspectives is characteristically dominant within particular healthcare stakeholder constituencies, they may be equated with the specific roles played by those parties. "Population health" may seem to be the property of insurers and government regulators, whose principal concerns are costs and the allocation of healthcare resources. Reciprocally, individualized patient care constitutes a domain that practitioners are prone to claim as their own. However, a simple equation of perspective with role breaks down once it is recognized that populations are comprised of individual people whose health interests are inevitably bound up with those of their fellow citizens and of the communities of which they are part. For example, when a local or regional government abdicates responsibility for water safety, children in the community may come down with serious and irreversible manifestations of lead toxicity and emphatically "individual" health problems may result [1]. Reciprocally, when parents within a community, whether based upon false information and ignorance or on their interpretations of religious dogma, decline to allow their children to receive routine childhood immunizations, herd immunity may break down and epidemics of infectious diseases such as measles, hemophilus influenza type B or even polio may emerge [2]. Furthermore, the perspectives of individual protagonists within the healthcare enterprise cannot be predicted with accuracy. For example, a leading figure in health economics today, Michael Porter, is a champion of the priority of individual patient valuation of health outcomes as the bed rock foundation of health economic theory and policy [3].

Michel Accad, an American internist and cardiologist in private practice in California, makes his perspective on the healthcare system crystal clear in the course of his book "Moving Mountains: A Socratic Challenge to the Theory and Practice of Population Medicine" (hereafter "Moving Mountains"), closing the volume with the thought: "... we can hope for the return of a more natural medicine, caring for patients one individual at a time" [2]. Accad has framed his impassioned defense of the domain of individualized patient care as a polemic against the concept of population health. The body of the book is set as a fantasied Socratic debate in which Accad plays both parts, that of the ancient Greek sage and also that of Geoffrey Rose, a British epidemiologist who died in 1993 and who is widely credited as the originator of the theoretical basis of the population health construct. Although it seems unlikely that the historical Socrates had a personal opinion on the issues of population health or of Rose's contributions, the Socrates of "Moving Mountains" is advantaged by knowledge of writings that appeared after Rose's demise and also seems to have well defined contemporary political views. A clinical focus, the definition and management of hypertension, pervades the volume and makes the arguments particularly salient today. Rose was a student of Sir George Pickering, whose treatise on blood pressure [5] embodied many of the principles that were subsequently generalized by his student as the premises of population health. 
The imaginary dialogue with Rose in "Moving Mountains" is sandwiched between an Introduction and an Epilogue, in which Accad avowedly voices his own views. Along the way, the reader finds some perceptive insights, particularly the observation that evidence-based medicine (EBM), as an ideology, can be seen as an attempt to subjugate the care of individual patients to populationbased findings [6]. Ultimately, Accad paints a uniformly dour picture of the population health movement and appears to conflate it with most of the toxicities that he perceives within the current climate of healthcare. He does not, however, identify or elaborate the specific ways that current concepts of population health and related programs impinge upon or impede the ability of a practitioner to care for her or his patients in a satisfying way. This is a substantial weakness of "Moving Mountains". As a result, much of the argument is unfocused and, at times, flailing. Furthermore, Accad's representation of Rose's views as presented in the latter's main writings $[7,8]$ is distorted. What makes Accad's book worthy of comment is its pertinence to current issues on the healthcare scene, particularly recent developments in the field of hypertension. It also highlights the need for precision in distinguishing between specific dimensions of scientific inquiry related to healthcare and the political interests with which they may transiently seem to cohere. The current review continues by successively addressing Rose's view of the interplay between population health and individual patient care, with application to the hypertension issue and, briefly, some of the societal issues raised by Rose and Accad.

\section{Geoffrey Rose and Population Health}

Accad's fantasy dialogue between a prestigious British epidemiologist and an ancient sage constitutes a novel vehicle and, at times, makes for entertaining reading. It renders the volume easily approachable by readers not otherwise familiar with such content. The book can be recommended to interested readers as a quick introduction to the issues surrounding the topic of population health that are potentially troublesome to professionals whose training and orientation has been devoted to the care of individual patients. Readers desiring more than a cursory introduction to those issues will need to appreciate that Accad's representation of Rose does not ultimately do justice to the latter's ideas. Specifically, what Accad denounces at the end of his introduction as a "confused hodge-podge of propositions that are untenable on clinical, epidemiological, social and ethical grounds" [4] is probably more accurately seen as Rose's attempt to elucidate the complexity underlying the issues of population versus individual health and the interplay of healthcare options, interventions and tradeoffs between individual and population health outcomes that need to be considered in the context of health policy development. To clarify the distinction between the Rose of his published writings and Rose as represented in "Moving Mountains", I will outline the former in some detail.
Although Rose's "Strategy for Preventive Medicine” [8] is his seminal work, the publication that perhaps most clearly presents the underlying kernel insight appeared in the International Journal of Epidemiology in 1985 [7]. The essential idea is straightforward. Standard approaches to the assessment of risk within a population involve statistical techniques such as logistic regression that are an accurate way of identifying factors within the population that increase or decrease the relative risk of acquiring or of suffering an outcome of a disease among individuals. However, such techniques fall short when it comes to identifying characteristics that are shared by all individuals within the population. The latter requires comparisons across societies, cultures or populations. The second part of Rose's insight is that the identification of risk factors that pertain to a population as a whole opens the door to consideration of classes of intervention that transcend the domain of the individual patient and may in fact not require that the beneficiaries be identified as "patients" at all, thereby side-stepping the de-personalizing aspects of much of healthcare in modern Society. Among such factors and potential interventions is a class of determinants that are not ordinarily considered to be "medical" and that lie outside of the domain of clinical practice to address. These are commonly called the "social determinants of heatlh" [9]. Rose's concept, however, applies no matter whether the determinants in question are properly "medical" or simply "social." The characteristics in question may or may not conform to a normal distribution curve within a target population. In fact, the essential concept does not require that the distributions be normal or even uni-modal. Rose's conjecture, based upon his mentor Pickering's insight regarding hypertension [5], is that population-based interventions might shift the distribution of such characteristics and the burden of consequent unwelcome health outcomes in a favorable direction. Hence the image of "Moving Mountains" employed by Accad as the title of his book [4].

Throughout his book [8], Rose appears intent on avoiding oversimplifying the interplay between the individual patient versus the population-based approaches. Rather, he sees advantages and disadvantages of both levels of intervention. For example, the individual patient, or "dichotomized" approach [8] selects individuals who, by virtue of their personal characteristics or severity of presentation, are at higher risk of adverse health outcomes, defines them as patients and recommends treatment. Much of the time this means therapy with pharmacological agents. This approach seeks a relatively high likelihood of individual benefit from such treatment. It also renders it relatively easy to monitor healthcare facilities and individual practitioners for compliance with recommendations and, reciprocally, easier for the latter to demonstrate such compliance. The principal disadvantage of the individual patient approach is that individuals with lower severity and lower risk of the unwanted outcomes are much less likely to benefit personally even though they are at the same risk of adverse treatment effects [8]. They also have to assume the role of "patients" with the consequent threats to personhood. At the same time, collectively, these lower risk individuals far outnumber those in the higher risk group. Consequently, it is people 
who are at lower personal risk who ultimately contribute the majority of unwanted outcomes within the population $[7,8]$. Hence, maximizing the likelihood of benefit for the individual patient works against the interests of population health. Furthermore, simply lowering the thresholds for recommended treatment results in converting many more individuals into "patients" and exposes them to adverse effects of treatment when in fact their absolute risks of such outcomes are very small. To the extent that a population-based intervention were available that could uniformly and collectively modify the relevant risk factor, then the adverse effects of treatment, the problem of "false positive” screening results and the unnecessary labeling of individuals as "patients" could all be avoided.

Rose, of course, is not unique in having identified the relative absence of individual incentive that accompanies adherence to recommendations that may be extremely beneficial on a population-based level. On 25 October 2017, The Times of London carried the following from a Letter to the Editor:

“... I am one of the 1.4 million people who should be on a statin, but isn't ('Doctors give statins to only one fifth of patients who qualify', News, Oct 24). When the National Institute for Health and Care Excellence recommended that I take the drug in 2014, I went to the NICE website to work out how much benefit I would gain. A year of statin treatment would prolong my life by 18 hours. Even before subtracting the time I would lose collecting repeat prescriptions, I decided it wasn't worth the bother" [8].

However, the population-based approach has its own disadvantages, as Rose elaborates in the later chapters of his treatise [8]. Firstly, there may not be a viable point of intervention capable of affecting the population as a whole in the desired fashion. Secondly, even if such exists, it may require coercive methods to successfully implement. Rose is clear in his rejection of the latter and advocates an approach in which individual choice is preserved. In other words, he seems to reject a "nanny state" approach to public health interventions. It is not clear where he would draw the line or how he would have responded to approaches such as those implemented in New York City a few years ago [11]. However, Rose recognized that both individual-based and population-based practice needed to co-exist in harmony. In Chapter 2 of his book he states:

"Disease truly forms a continuum of severity, but its management requires a system of unambiguous labels. The big mistake has been, not the use of dichotomous diagnostics, but to consider that process as being a description of the natural order rather than merely an operational convenience. Management policy requires 'yes/no' decisions such as investigate or not investigate, admit or send home, treat or not treat ... To improve the nation's health statistics for consultations, hospital admissions, and mortality is indeed an important measure of preventive success, but it is not sufficient for it fails to consider that much of the population's burden of ill health derives from a mass of less obvious troubles which doctors do not see. For example, in our studies of angina we found that only about a quarter of all the sufferers had ever been diagnosed. There is a vast submerged burden of ill health” [8].

Returning to the Rose of "Moving Mountains" [4], Accad lends a semblance of authenticity of his representation by interspersing the text with cited direct quotations from Rose's writings. However, the excerpts are selected and Rose's actual views are distorted. Accad seems bent on the portrayal of the population health concept as adversarial to the care of individual patients and chooses to interpret Rose's articulate acknowledgement of the complexity of the issues as evidence of "a confused hodge-podge of propositions”, ignoring the actual thread of Rose's argument. Indeed, a glaring example of Accad's distortion of Rose occurs in connection with the principle of informed individual choice and the highly related construct of shared decision-making [12]. In his Chapter 5, Accad (as both Socrates and Rose) portrays Rose as a traditional, paternalistic, prescriber of what is "good for" the individual patient and as an adversary of shared decision-making [4]. However, in Chapter 8 of his treatise, Rose himself pronounces:

\begin{abstract}
"The treatment is offered, and individuals can then decide for themselves whether or not they wish to take it. Nothing is imposed and no one is compelled. This applies whenever the potential recipients are free to accept or reject the advice, whether it be on fluoridated toothpaste, diet, or smoking. This freedom of choice is only effective if those concerned have access to all the relevant information and are able to understand it. In practice, access to relevant information may be difficult or impossible to obtain because those who control its supply may not wish or be able to share it fully or to present it in a neutral, value-free way. Physicians and their teams present information so as to favour what they see as the right choice, and the news media present it so as to favour their editorial policy or to make a good story” [8].
\end{abstract}

It would seem that Accad's distortion of Rose is not the arbitrary result of hasty reading or of academic inexperience, but rather a systematic attempt to portray Rose as the mother of evil behind an ongoing quest to "advance population medicine as a replacement for traditional patient care” as voiced by Accad on page 2 of his Introduction [4].

\section{The Case of Hypertension}

The issues raised by Rose and more indirectly by Accad's critical dialogue [4], are rendered particularly salient by recent developments in the field of hypertension definition and management. Much of Rose's theory of population health was founded on a proposition introduced by his teacher, Sir George Pickering, that blood pressure values are continuously distributed across the population [5]. If this were the case, then the establishment of a dichotomous cut-off above which pressures would be considered to be "abnormal" and subject to modification for purposes of 
diminishing cardiovascular risk, would be arbitrary and necessarily a matter of "operational convenience", rather than a physiologically defined value [8]. The lower the threshold value, the larger percentage of the population is arbitrarily defined as "diseased" and the lower the efficiency of treatment for the individual. On the other hand, if interventions could be identified that could be applied to the entire population, efficiency would no longer be defined on an individual patient basis [8]

Recent developments within the US hypertension guideline community bear directly on these issues. Over the last two decades, the recommended treatment threshold level for blood pressure elevation advanced by the US Joint National Committee on Hypertension and by the American Heart Association (AHA) in conjunction with the American College of Cardiology (ACC) has been gradually decreasing. The latest update of the AHA/ACC guidelines, released in 2017, recommends a lowering of the threshold for defining treatable blood pressure elevation from $140 \mathrm{~mm} \mathrm{Hg}$ to $130 \mathrm{~mm} \mathrm{Hg}$ systolic [13]. As a result, relative to the previous recommendations, an additional $13 \%$ of US adults, or a total of just over $45 \%$, will be defined as hypertensives [11]. However, only an additional $2 \%$ of the population will be recommended to consider drug therapy based upon increased risk factors for cardiovascular disease. This likely reflects concern for increased side effects of treatment which can include hypotension and syncope. Such effects were observed in the SPRINT trial upon which the new recommendations are largely based [14].

The magnitude of potential benefit for individuals whose blood pressures lie in the lower range of the treatment eligible group is small. The SPRINT trial enrolled non-diabetic participants whose cardiovascular risk was calculated to be high [14]. The magnitude of benefit for those receiving active treatment was about half of a percent lower likelihood of an important cardiovascular outcome per year. The benefit for individuals lacking such risk factors would be only $3 \%$ over a 10 year period $[15,16]$. This means that, on average, 33 low risk people would have to be treated, pharmacologically or otherwise, for 10 years for a single person to avoid an event. The new hypertension recommendations also have important implications for individuals with higher pressures who are already under treatment. Since many of them, even with treatment, have pressures outside the new threshold range, they will need more intensive therapy and more drugs and will risk more serious adverse effects of treatment [15]. It would seem that the consequences of trends in recommendations for defining and managing hypertension well illustrate the principles elaborated by Rose [8]. That is, the lower the threshold for defining individual "disease", the greater the shift from individual to population level benefit $[15,16]$. When the threshold is lowered, more cardiovascular events are avoided at the expense of greater numbers of individuals being defined as "diseased" and being treated with minimal individual health benefit and despite the same risk of adverse events from treatment. As an advocate of population-based interventions, it would seem that Rose might well be viewed as a champion of personcentered healthcare. That is, for purposes of maximizing health outcomes on a population level, Rose favored population level interventions, minimizing the need to define individual persons as "diseased patients".

Although Accad quibbles with some of Pickering's original assumptions [5] based on a paper published in 1983 [17], subsequent clinical research seems to have consistently born out Rose's projections. To be sure, an effective approach to treatment of hypertension, as a population level "disease", rather than as an individual ailment, is yet to be identified. However, Rose was not in his writings claiming to have found magic bullets or perfect solutions. Rather, he seems to have been attempting to characterize the complexity of health problems such as hypertension and in so doing to clearly differentiate between disease as we conventionally understand it on an individual level and potentially treatable characteristics on a population level. From thirty years of clinical experience as an emergency physician, this reviewer can attest that confusion regarding hypertension as a primary clinical entity versus as a predictor of clinical outcomes has been and remains rampant in the emergency room. Specifically, the practice of acutely lowering elevated blood pressures (which in the ER commonly reflect a normal physiological response to the stress of being in that environment), although consistently discouraged in published guidelines, has proved very difficult to curtail. Indeed, for over 20 years the practice has been known to cause harm $[18,19]$. Its persistence appears to reflect health professionals' conceptual difficulty in grasping Rose's principal point within an acute care setting.

\section{Some Additional Considerations and Conclusion}

"Moving Mountains" is written for a middle level readership and, despite its limitations, provides a very approachable vehicle for readers such as clinicians and interested health consumers to gain an initial appreciation of the nature of the issues. However, readers should consult original sources if they wish to gain a fully informed understanding of the relevant perspectives. Readers should be able to detect for themselves the somewhat idiosyncratic, if impassioned, nature of Accad's views on matters of ethics and social justice. For example, in the epilogue, he implies that the American College of Physicians Ethics, Professionalism and Human Rights Committee is a mere tool of health policy makers and the insurance industry [4]. Accad cites the following as evidence thereof:

\footnotetext{
"Resource allocation decisions are most appropriately made at the policy level rather than entirely in the context of an individual patient-physician encounter. Ethical allocation policy is best achieved when all affected parties discuss what resources exist, to what extent they are limited, what costs attach to various benefits, and how to equitably balance all these factors" [20].
}

Considering that new drugs are being introduced into the marketplace at a price of half a million US dollars per 
dose [21], Accad's implied suggestion that the insurance industry should be excluded from deliberations about allocations of healthcare resources seems out of touch. Accad's responses to the non-medical side of Rose's original thesis verge on flailing. He apparently regards recognition of social, economic and environmental factors as health co-determinants and the importance of addressing disparities in the quality of delivered healthcare to be tantamount to an ultra-left wing political agenda. In the body of the dialogue Accad, as Socrates, all but brands Accad, as Rose, a communist, referring to his treatise as "The Little Rose Book" [4]. In the Epilogue, he equates the principles of population health to a utopian, "egalitarian" world view. Quite apart from matters of personal political perspective, it seems unclear why eliminating disparities in health and living conditions and extending healthcare access to the entire population would not be favored by health professionals as a natural consequence of their role in Society. This aspect of Accad's perspective is not explained in his book.

For reasons that are poorly substantiated in "Moving Mountains", Accad has settled on the population health construct as the principal source of evil within today's US healthcare system. Of course, one might find it refreshing to find that something other than evidence-based medicine (EBM) has been placed in this role. (Published perspectives on the latter have suggested that "EBM" might better be understood as an abbreviation for either "Everything Bad in Medicine" [22] or, in a reborn mode, as "Everything Best in Medicine” [23] ). As previously noted, Accad observes that EBM, as "evidence-based medicine”, insofar as it has attempted to subjugate clinical decision-making to findings drawn from studies on research populations, could be seen as a special case of infringement of population-based principles into the domain of clinical practice. Although somewhat metaphorical, this notion ranks as a highlight of his book and is appropriately referenced to the published literature [6]. However, ultimately, the attempt to find a single, convictable culprit behind the toxicities of what has become in the US a multi-trillion dollar per year healthcare enterprise, is likely destined to generate only confusion and consternation and to distract from the kind of concerted and coordinated efforts required to create a dent in the complexity of the problems.

All this said, the degree of consternation among American private medical practitioners in recent years should not be under-estimated or dismissed in importance. Piled on demands for "documentation", endless piles of forms and applications, be they electronic or paper, mandated electronic medical record systems that do not work, the constant threat of lawsuits and the defensive necessities to avert them, required authorizations, insurance denials requiring resubmitted requests, the list goes on and on, collectively exerting enormous pressure. Within this maelstrom, insurance companies and government regulators constitute conspicuous targets. Michel Accad's perspective certainly reflects the pressures that practitioners in such systems are experiencing, as does his expressed yearning to return to an idyllic time during which practitioners and patients could conduct their affairs within a truly private practice setting. Accad is exceptional insofar as he has taken the time to put thoughts on paper that pertain to the dilemmas in which he and his colleagues find themselves. Readers may gain an initial, if distorted, picture of what the population health issue is all about and may appreciate something about the context which gave rise to the narrative. They may also hope that the author considers developing a more direct elaboration of the nature of the threats to the care of individual patients that he perceives and of how they relate to the various elements at play within today's healthcare environment.

\section{Conflicts of Interest}

The reviewer declares no conflicts of interest.

\section{References}

[1] https://en.wikipedia.org/wiki/Flint_water_crisis.

[2] https://www.cdc.gov/vaccinesafety/concerns/autism.html.

[3] Porter, M.E. (2010). What Is Value in Health Care? New England Journal of Medicine 363, 2477-2481.

[4] Accad, M. (2017). Moving Mountains: A Socratic Challenge to the Theory and Practice of Population Medicine. Huntsville, TX: Green Publishing House LLC. [5] Pickering, G. (1968). High Blood Pressure, 2nd Edition. London: J \& A Churchill.

[6] Tanenbaum, S.J. (2014). Particularism in health care: challenging the authority of the aggregate. Journal of Evaluation in Clinical Practice 20, 934-941.

[7] Rose, G. (1985). Sick Individuals and Sick Populations. International Journal of Epidemiology 14 (1) 32-38.

[8] Rose, G. (2008). Rose's Strategy of Preventive Medicine 2nd Edition. New York: Oxford University Press.

[9] Mechanic, D. (2007). Population Health: Challenges for Science and Society. Milbank Quarterly 85 (3) 533559.

[10] Letter to the Editor. (2017). Stalling On Statins. In: The Times, London 25 October 2017, p.26.

[11] https://gizmodo.com/the-complete-list-of-everythingbanned-by-mayor-michael-1490476691.

[12] Elwyn, G., Tilburt, J. \& Montori, V.W. (2013). The ethical imperative for shared decision-making. European Journal for Person Centered Healthcare 1 (1) 129-131.

[13] Whelton, P.K., Carey, R.M., Aronow, W.S., et al. (2018). 2017 ACC / AHA / AAPA / ABC / ACPM / AGS / APhA / ASH / ASPC/ NMA / PCNA Guideline for the Prevention, Detection, Evaluation, and Management of High Blood Pressure in Adults: A Report of the American College of Cardiology/American Heart Association Task Force on Clinical Practice Guidelines. Journal of the American College of Cardiology 71 (19) e127-e248. Eratum: Journal of the American College of Cardiology 71 (19) 2275-2279.

[14] Wright, J.T., Williamson, J.D., Whelton, P.K., Snyder, J.K., Sink, K.M., Rocco, M.V., Reboussin, D.M. \& Rahman, M. (2015). A Randomized Trial of Intensive 
versus Standard Blood-Pressure Control. New England Journal of Medicine 373 (12) 2103-2016.

[15] Muntner, P., Carey, R.M., Gidding, S., Jones, D.W., Taler, S.J., Wright, J.T. \& Whelton, P.K. (2018). Potential U.S. Population Impact of the 2017 American College of Cardiology/American Heart Association High Blood Pressure Guideline. Circulation 137 (2) 109-118.

[16] Muntner, P. \& Whelton, P.K. (2017). Using Predicted Cardiovascular Disease Risk in Conjunction With Blood Pressure to Guide Antihypertensive Medication Treatment. Journal of the American College of Cardiology 69 (19) 2446-2456.

[17] McManus, I.C. (1983). Bimodality of Blood Pressure Levels. Statistics in Medicine 2, 253-258.

[18] Grossman, E., Messerli, F.H., Grodzicki, T. \& Kowey, P. (1996). Should a Moratorium Be Placed on Sublingual Nifedipine Capsules Given for Hypertensive Emergencies and Pseudoemergencies? Journal of the American Medical Association 276 (16) 1328-1331.

[19] Winker, M. (1996). The FDA's Decisions Regarding New Indications for Approved Drugs Where's the Evidence? (Editorial). Journal of the American Medical Association 276 (16) 1342-1343.

[20] Snyder, L. (2012). American College of Physicians Ethics Manual, Sixth Edition. Annals of Internal Medicine 156 (1 Pt2) 73-104.

[21] http://www.onclive.com/web-exclusives/novartis-setsa-price-of-475000-for-car-tcell-therapy.

[22] Wyer, P.C. \& Silva, S.A. (2013). Tarnished Gold: The Sickness of Evidence-based Medicine by Steve Hickey and Hilary Roberts: EBM as an Avatar of Modern Medicine. European Journal for Person Centered Healthcare 1, 8087.

[23] Wyer, P. \& Silva, S.A. (2015). 'All the King's horses . . .': the problematical fate of born-again evidence-based medicine: commentary on Greenhalgh, T., Snow, R., Ryan, S., Rees, S. and Salisbury, H. (2015) six 'biases' against patients and carers in evidence-based medicine. BioMed Central Medicine 13, 200. Journal of Evaluation in Clinical Practice 21 (6) E1-E10. 